Research Article

\title{
Modeling and Experimental Study on Dynamic Characteristics of Dual-Mass Flywheel Torsional Damper
}

\author{
Long Chen (iD, Wen-ku Shi (D), and Zhi-yong Chen (i) \\ State Key Laboratory of Automotive Simulation and Control, Jilin University, Changchun 130022, China \\ Correspondence should be addressed to Zhi-yong Chen; chen_zy@jlu.edu.cn
}

Received 8 January 2019; Accepted 15 April 2019; Published 13 May 2019

Academic Editor: Athanasios Chasalevris

Copyright (C) 2019 Long Chen et al. This is an open access article distributed under the Creative Commons Attribution License, which permits unrestricted use, distribution, and reproduction in any medium, provided the original work is properly cited.

Theoretical modeling and experimental research are carried out on the dynamic torsional characteristics of a dual-mass flywheel (DMF) in this paper. Firstly, the structure and working principle of the DMF are analyzed. Secondly, the arc spring is analyzed by the discrete element method. For the different frictional torques in the working process, the linear fitting and equivalent energy methods are used to model the frictional torque under the dynamic condition of the arc spring. The fractional derivative model is used to model the viscous damping of DMF. Then, the parameter identification and model verification are carried out on the model, and the model error is analyzed. Finally, an experimental study on the dynamic torsional characteristics of DMF is carried out. The results demonstrate that the torsional stiffness of the DMF varies with the excitation amplitude and frequency. This modeling and test method can be used for structural design and performance prediction analysis of DMF.

\section{Introduction}

Torsional vibration of an internal combustion engine vehicle powertrain is another major source of vibration excitation for vehicles in addition to road excitation. The torsional vibration of the powertrain is mainly caused by the cyclical change of the engine cylinder pressure and the inertial force generated by the reciprocating motion of the crank-link mechanism, which causes the engine output torque to fluctuate and brings torsional vibration. The application of the elastic torsional vibration damper mounted on the clutch disc reduces the torsional vibration of the powertrain, but due to the limited space of the clutch torsion damper spring, the torsion angle is small, the torsional stiffness is large, and the effect of vibration reduction is limited $[1,2]$. Moreover, the clutch torsional vibration damper cannot reduce the resonance speed of the powertrain below the idle speed so that the transmission system still has the possibility of resonance in the common speed range of the automobile. Although the promotion and application of high-power and lightweight engines have achieved the goal of improving vehicle power and reducing emissions, this has also led to more severe torsional vibration of automobile transmission systems, which has become an urgent problem for traditional internal combustion engine vehicles to improve $\mathrm{NVH}$ performance of complete vehicles. As a new type of the torsional vibration damper, the $\mathrm{DMF}$ has a large torsion angle and a small torsional stiffness compared to the clutch torsional vibration damper so that the speed corresponding to the natural frequency of the powertrain is much lower than the idle speed. Therefore, the powertrain does not resonate within the normal speed range of the engine, reducing the torsional vibration of the powertrain and improving the ride comfort of the vehicle [3].

At present, the DMF plays a very important role in the vibration reduction, noise reduction of the powertrain, solving the problem of engine knocking, improving the smoothness of the shift, and so on. At the same time, there are many academic researches on the dual-mass flywheel. Kim used the discrete element method to analyze the effect of the friction on the arc spring on torsional performance of the DMF [4]; Tom conducted theoretical and experimental research on the torsional characteristics of long arc spring DMF and estimated the engine output torque using DMF [5]. Through the experimental study on the torsional characteristics of the DMF, Chen established a nonlinear 
dynamic model of the DMF based on the viscous damping [6]; Song proposed a DMF with continuous variable stiffness by using shape constraints and conducted theoretical and experimental studies on its torsional characteristics [7, 8]. $\mathrm{Zu}$ et al. proposed an intelligent magnetorheological DMF, which can realize the real-time adjustment of the damping coefficient to achieve semiactive control of the torsional vibration of the powertrain [9]; Tang et al. studied the effect of dual-mass flywheels on the torsional vibration performance of hybrid electric vehicles and proposed a motor torque control methods to suppress the torsional vibration [10-12]. The existing literature has carried out a lot of research on the structure of the DMF, torsional characteristics, and vibration damping performance, but it can be found that there are few studies on the dynamic torsional characteristics (dynamic stiffness and lag angle) of the DMF. The dynamic torsional characteristics of the DMF directly determine its damping performance, and the test results also show that the performance will change greatly under dynamic torsion. Based on the existing literature, the dynamic torsion characteristics of DMF are modeled and tested in this paper.

This paper is organized as follows: Section 1 is devoted to describing the composition and working principle of the DMF; Section 2, the dynamic characteristics of DMF are modeled. In Section 3, the parameter identification and experimental verification of the dynamic characteristic model built in Section 2 are carried out, and the model error is analyzed. In Section 4, the dynamic characteristics of DMF are experimentally studied. Section 5 summarizes the conclusions of this paper.

\section{DMF Structural Principle}

The structure of the DMF is shown in Figure 1. It is composed of the first mass, the second mass, the starting ring gear, the force transfer plate, and the long arc spring [13]. The starting ring gear is connected to the first mass by an interference fit. The first mass is bolted to the end flange of the engine crankshaft. The flange is connected to the second mass by rivets. The second mass is bolted to the clutch assembly; the first mass assembly and the second mass assembly are connected by a low stiffness arc spring and can rotate relatively. When the engine crankshaft rotates, it drives the first mass to compress the arc spring through the boss. The other end of the arc spring drives the ears on both sides of the transmission plate, thus driving the second mass to rotate and realizing the transmission of power from the engine to the gearbox.

The DMF structure solves the shortcomings of the traditional clutch torsional vibration damper spring, such as small torsional angle and large stiffness. Moreover, the moment of inertia of the first mass and the second mass can be flexibly distributed so that the resonance speed of the powertrain is kept away from the operating speed of the engine. Also the torque fluctuation of the input shaft of the transmission and the shifting noise are greatly reduced, and the shift smoothness and the NVH performance of the vehicle can be improved $[14,15]$.

\section{Dynamic Characteristic Modeling}

The arc spring is the key component of the DMF structure. The performance of the arc spring determines the torsional vibration reduction performance of the DMF. Therefore, the arc spring is first analyzed and modeled in this paper.

3.1. Arc Spring Frictional Torque Modeling. The frictional torque is generated by using the arc spring and the sheath during the working process which is composed of two parts, as shown in Figure 2. The first part is that as the torsion angle $\theta$ increases, it produces a radial positive pressure along the radial direction of the distribution, thereby generating a frictional torque during the arc spring is compressed by the torque $T$; the other part is that the arc spring is subjected to centrifugal force to generate positive pressure on the sheath, which generates frictional torque when the speed of DMF is $\Omega$.

3.1.1. Frictional Torque Generated by Radial Component. The discrete element method is used to analyze the influence of arc spring on the dynamic characteristics of DMF. The total mass of arc spring is $m$, which is divided into $n$ units. Each unit has a mass of $m_{i}(i=1,2,3, \ldots, n)$ and each element stiffness is $k_{1}=k_{2}=\cdots=k_{n} ; c_{i}$ is the arc spring damping coefficient [4]. Arc spring is shown in Figure 3(a). The equivalent model of the discrete arc spring is shown in Figure $3(\mathrm{~b})$, and the angle between the elements is $\theta_{i}$.

Firstly, the quasi-static compression is analyzed (without considering the frictional torque generated by centrifugal force). The $k$-th arc spring mass element is used to analyze the stress state of the arc spring, as shown in Figure $4 . F_{k-1}$ is the force of the last spring mass element, $F_{k}$ is the force of the next spring mass element, and $F_{\mathrm{F}}$ is the tangential friction force of the slide way. $F_{0}$ is the radial force, $F_{N}$ is the support force of the slide to the arc spring, $R$ is the distribution radius of the arc spring, $\mu$ is the friction coefficient, and $k_{i}$ is the stiffness coefficient.

The arc spring is loaded clockwise, and the force is decomposed in the normal and tangential directions. According to the force balance, the following equation is obtained.

Tangential direction:

$$
m_{i} R \dot{\omega}_{k}=\cos \left(\frac{\theta_{k}-\theta_{k-1}}{2}\right) F_{k-1}-\cos \left(\frac{\theta_{k+1}-\theta_{k}}{2}\right) F_{k}-F_{\mathrm{F}} .
$$

Normal direction:

$$
m_{i} R \omega_{k}^{2}=-\sin \left(\frac{\theta_{k}-\theta_{k-1}}{2}\right) F_{k-1}-\sin \left(\frac{\theta_{k+1}-\theta_{k}}{2}\right) F_{k}+F_{N},
$$

where $\omega_{k}$ is the angular velocity of the mass element, which can be approximated to zero due to quasi-static loading. According to formula (2), the following equation is obtained: 


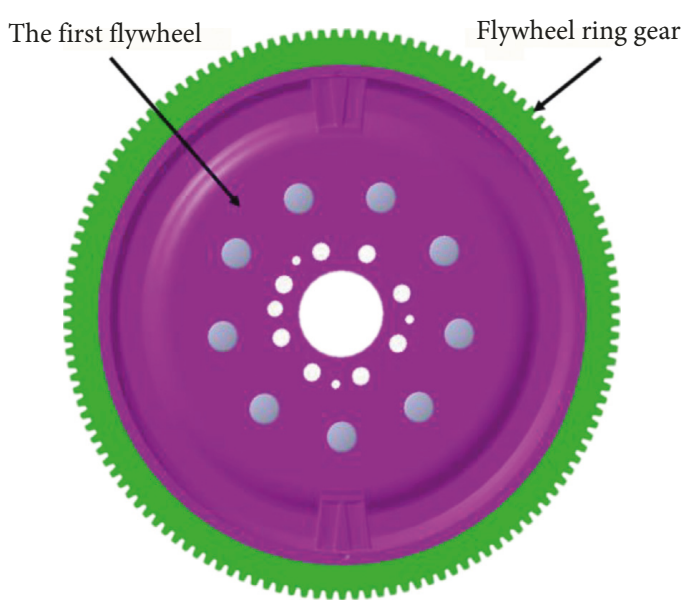

(a)

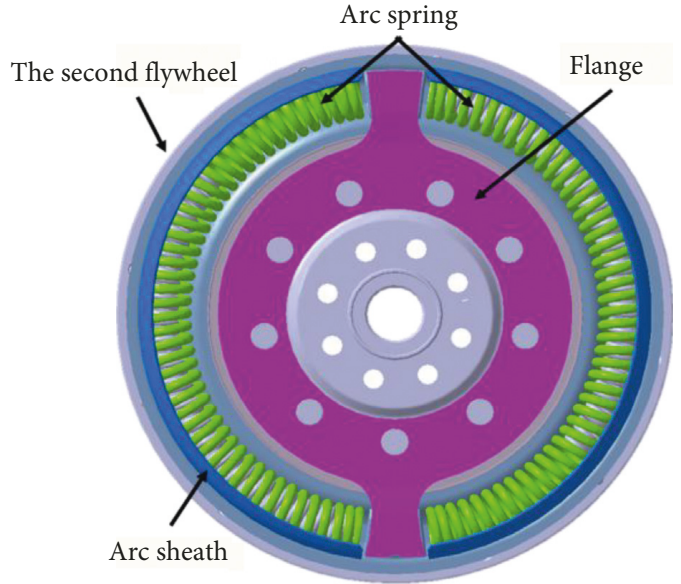

(b)

Figure 1: Arc spring DMF.

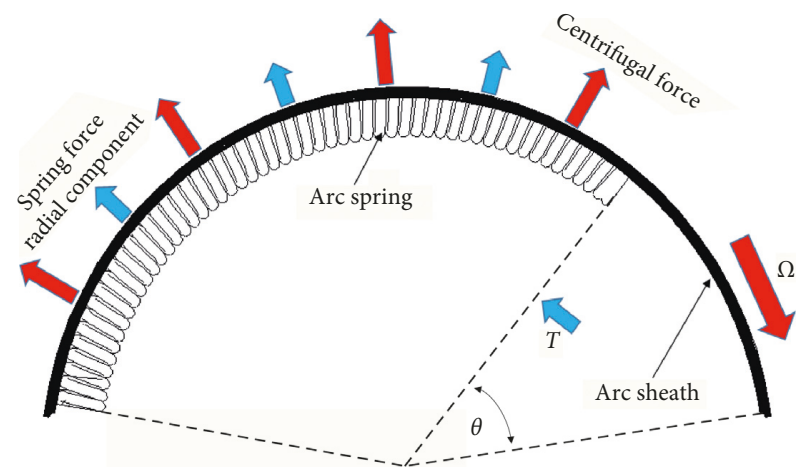

FiguRe 2: Diagram of frictional moment of arc spring.

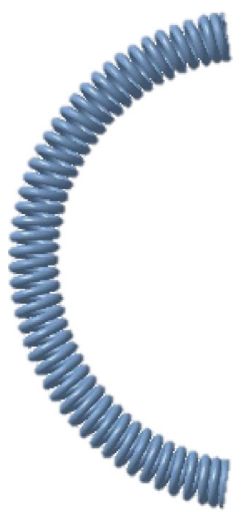

(a)

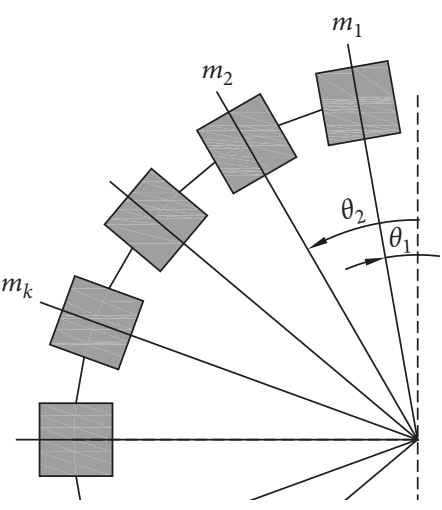

(b)

Figure 3: Structural diagram of (a) arc spring and (b) discrete element.

$$
F_{N}=\sin \left(\frac{\theta_{k}-\theta_{k-1}}{2}\right) F_{k-1}+\sin \left(\frac{\theta_{k+1}-\theta_{k}}{2}\right) F_{k}
$$

The tangential friction force is

$$
F_{\mathrm{F}}=\mu F_{N}
$$

The angle difference of any two elements is

$$
\varphi_{i}=\frac{\alpha}{n}-\frac{F_{i}}{k_{i} n}
$$

where $\alpha$ is the central angle of the arc spring's free length and $n$ is the number of arc spring elements.

The tangential force formula of the $i$-th element can be obtained: 


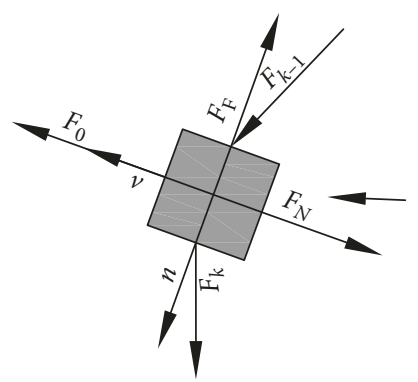

FIgURE 4: Force diagram of the arc spring element.

$$
F_{k}=\frac{\left(\cos \varphi_{i}-\mu \sin \varphi_{i}\right) F_{k-1}}{\cos \varphi_{i}+\mu \sin \varphi_{i}}
$$

When unloading, except for the reverse friction force, other forces are the same as those loading. So the tangential force formula of the $i$-th element can be obtained:

$$
F_{k}=\frac{\left(\cos \varphi_{i}+\mu \sin \varphi_{i}\right) F_{k-1}}{\cos \varphi_{i}-\mu \sin \varphi_{i}} .
$$

According to formulas (6) and (7), the relationship between the friction spring torque and the torsion angle of the arc spring under quasi-static loading conditions (without considering the influence of centrifugal force) can be obtained, as shown in Figure 5.

Figure 6 shows that the frictional torque generated is in a quasi-linear relationship with the torsion angle, and the relationship is obtained by linear fitting:

$$
T_{\mathrm{f} 1}=p_{1} \theta
$$

where $p_{1}=0.5619$ is the fitting coefficient. The fitting results are shown in Figure 6.

\subsubsection{Frictional Torque Generated by Centrifugal Force.} The frictional torque $T_{\mathrm{f} 2}$ generated by the centrifugal force of the arc spring is

$$
T_{\mathrm{f} 2}=\mu m R^{2} \Omega^{2} .
$$

When the speed of DMF is constant, the frictional torque generated by the centrifugal force is constant. The equivalent energy method can be used to calculate the equivalent damping coefficient under periodic excitation [16]. The equivalent damping coefficient can be simplified as

$$
c_{\mathrm{ef} 2}=\frac{4 T_{\mathrm{f} 2}}{\pi \omega \theta}=\frac{4 \mu m R^{2} \Omega^{2}}{\pi \omega \theta} .
$$

Finally, the total frictional torque of arc spring is obtained as follows:

$$
T_{\mathrm{f}}=T_{\mathrm{f} 1}+T_{\mathrm{f} 2}
$$

3.2. Equivalent Model of Arc Spring. According to Figure 3, the dynamic equation of the $i$-th element of the curved spring is obtained by Newton's second law:

$$
m_{i} R^{2} \ddot{\theta}_{i}+c_{i} \dot{\theta}+k_{i}\left(\theta_{i}-\theta_{i+1}\right)+T_{\mathrm{f} i}=k_{i-1}\left(\theta_{i-1}-\theta_{i}\right) .
$$

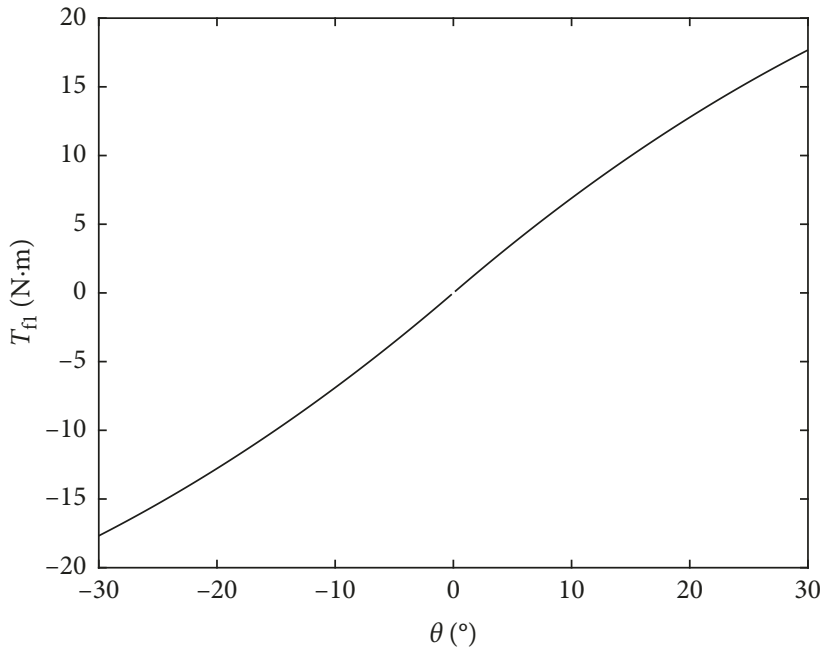

Figure 5: The relationship curve of frictional torque and torsion angle.

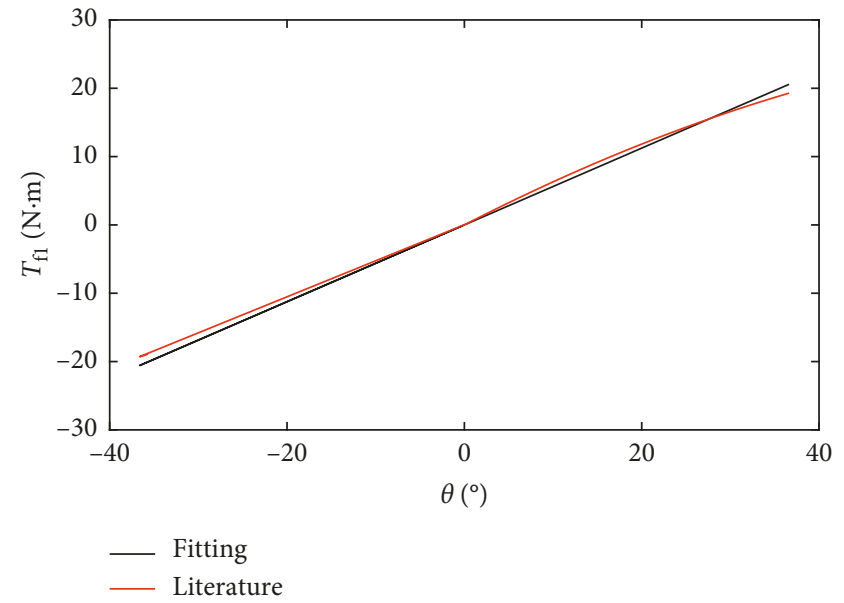

FIgURe 6: Fitting curve.

Thus, the mechanical equation of the whole arc spring can be obtained:

$$
\left\{\begin{array}{l}
m_{1} R^{2} \ddot{\theta}_{1}+c_{1} \dot{\theta}_{1}+k_{1}\left(\theta_{1}-\theta_{2}\right)+T_{\mathrm{f}}^{1}=k_{0}\left(\theta-\theta_{1}\right), \\
m_{2} R^{2} \ddot{\theta}_{2}+c_{2} \dot{\theta}_{2}+k_{2}\left(\theta_{2}-\theta_{3}\right)+T_{\mathrm{f}}^{2}=k_{1}\left(\theta_{1}-\theta_{2}\right), \\
\vdots \\
m_{n} R^{2} \ddot{\theta}_{n}+c_{n} \dot{\theta}_{n}+k_{n} \theta_{n}+T_{\mathrm{f}}^{n}=k_{n-1}\left(\theta_{n-1}-\theta_{n}\right) .
\end{array}\right.
$$

Based on the assumptions of discrete elements,

$$
\begin{aligned}
m_{1} & =m_{2}=\cdots=m_{n}=\frac{1}{n} m, \\
c_{1} & =c_{2}=\cdots=c_{n}=c, \\
k_{1} & =k_{2}=\cdots=k_{n}=n k, \\
T_{\mathrm{f}}^{1}+T_{\mathrm{f}}^{2}+\cdots+T_{\mathrm{f}}^{n} & =T_{\mathrm{f}} .
\end{aligned}
$$


Simplification of equation (13) can be obtained as follows:

$$
\sum_{i=1}^{n} m_{i} R^{2} \ddot{\theta}_{i}+c \sum_{i=1}^{n} \dot{\theta}_{i}+n k\left(\theta_{1}+\theta_{n}-\theta\right)+\sum_{i=1}^{n} T_{\mathrm{f}}^{i}=0 .
$$

In order to simplify the calculation, the arc spring is equivalent to a concentrated mass, and formula (15) can be simplified as follows:

$$
m_{\mathrm{e}} R^{2} \ddot{\theta}^{\prime}+c_{\mathrm{e}} \dot{\theta}^{\prime}+k_{\mathrm{e}}\left(2 \theta^{\prime}-\theta\right)+T_{\mathrm{f}}=0,
$$

where $m_{\mathrm{e}}$ is the equivalent mass, $c_{\mathrm{e}}$ is the equivalent damping, $k_{\mathrm{e}}$ is the equivalent stiffness, and $\theta^{\prime}$ is the equivalent mass torsion angle.

3.3. DMF Dynamics Modeling. Figure 7 is a simplified model of DMF, where $M_{0}$ is the frictional damping produced by the friction disc. According to the simplified model of Figure 7, the mathematical relationship expression of DMF is obtained:

$$
T=T_{\mathrm{s}}+T_{\text {fri }}+T_{\mathrm{d}}
$$

where $T_{\mathrm{s}}$ is the recovery torque generated by using the arc spring, $T_{\text {fri }}$ is the frictional torque, $T_{\mathrm{d}}$ is the viscous damping torque, and $T$ is the combined moment of the DMF.

The arc spring has complex viscous lubrication in the dynamic working process. In order to explain this complicated dynamic process, the fractional derivative order model is adopted in this paper [17]:

$$
T_{\mathrm{d}}=b D^{\beta}(\theta)+c \dot{\theta},
$$

where $b$ is the viscoelastic torque coefficient, $\beta$ is the fractional derivative order, $D^{\beta}(\cdot)$ is the $\beta$-order derivative of $(\cdot)$, and $c$ is the flywheel viscous damping coefficient.

The frictional torque generated by using the friction disc is mainly used to reduce the resonance peak, and its expression is as follows:

$$
T_{\text {fri }}=M_{0} .
$$

The equivalent damping coefficient is calculated by the equivalent energy method:

$$
c_{\mathrm{eq}}=\frac{4 M_{0}}{\pi \omega \theta},
$$

where $\omega$ is the excitation frequency.

The elastic recovery torque of DMF can be obtained according to Figure 7 as

$$
T_{\mathrm{s}}=k_{\mathrm{e}}\left(\theta-\theta^{\prime}\right) .
$$

In summary, the resulting torque of the DMF model is given by the sum of all element torques:

$$
\left\{\begin{array}{l}
T=b D^{\beta}(\theta)+\left(c+c_{\mathrm{eq}}\right) \dot{\theta}+k_{\mathrm{e}}\left(\theta-\theta^{\prime}\right), \\
m_{\mathrm{e}} R^{2} \ddot{\theta}^{\prime}+c_{\mathrm{e}} \dot{\theta}^{\prime}+k_{\mathrm{e}}\left(2 \theta^{\prime}-\theta\right)+c_{\mathrm{ef} 2} \dot{\theta}^{\prime}+p_{1} \theta=0 .
\end{array}\right.
$$

The Laplace transform is applied to equation (22), and the dynamic torsion complex stiffness of DMF can be simplified:

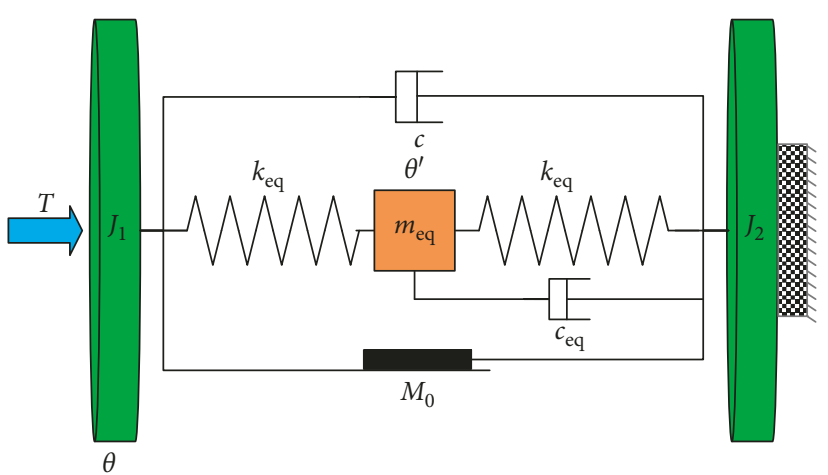

Figure 7: DMF simplified model.

$$
\begin{aligned}
K^{*}= & \frac{T(s)}{\theta(s)}=b s^{\beta}+\left(c+c_{\mathrm{eq}}\right) s \\
& +k_{\mathrm{e}}\left(1-\frac{k_{\mathrm{e}}}{m_{\mathrm{e}} R^{2} s^{2}+\left(c_{\mathrm{e}}+c_{\mathrm{ef} 2}\right) s+2 k_{\mathrm{e}}+p_{1}}\right) .
\end{aligned}
$$

\section{Parameter Identification and Experimental Verification}

In this section, the dynamic characteristics of the DMF are tested, and then the dynamic stiffness curve of the DMF is obtained by the geometric drawing method. The parameters of the model established in the previous section are identified and validated.

\subsection{Test Equipment and Scheme}

4.1.1. Test Device. A SCHENCK electro-hydraulic servo torsion test rig is used to test the dynamic torsion characteristics of DMF, as shown in Figures 8 and 9. Properties of the equipment are as follows: torque range is $0 \sim 1000 \mathrm{~N} \cdot \mathrm{m}$, torsion frequency range is $0 \sim 100 \mathrm{~Hz}$, and torsion angle can be $0 \sim 50^{\circ}$. The DMF parameters in this paper are shown in Table 1.

The second mass of the DMF is connected to the fixed bracket through the clamp. The first mass is connected with the torsion actuator. The test bench is equipped with an angular displacement sensor and a torque sensor. The angular displacement and the torque signal are collected in real time by using the data acquisition device. The torsion characteristic curve of the DMF is obtained. Then the data are processed to obtain a dynamic mass characteristic curve of the DMF.

4.1.2. Test Loading Scheme. The loading scheme of the DMF dynamic stiffness test is as follows:

(i) Dynamic stiffness excitation can be expressed by the following formula:

$$
\theta=\Theta+\theta_{0} \sin \omega t
$$

where $\Theta$ is the pretwisted angle and $\theta_{0}$ is the magnitude of the excitation torsion angle. 


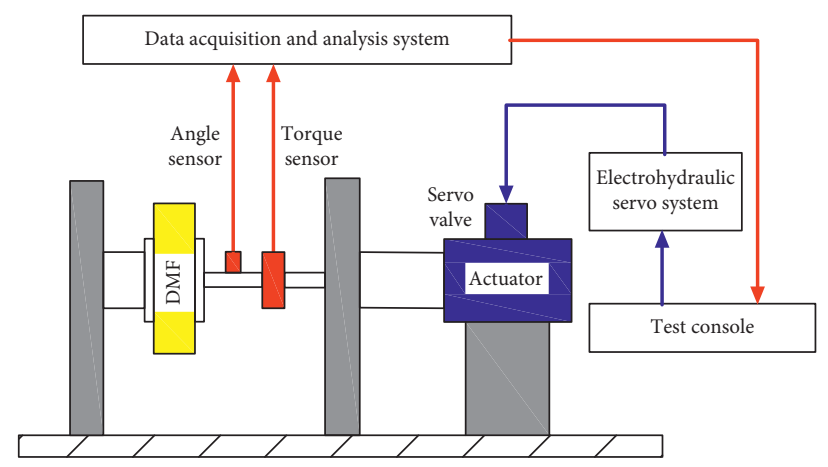

FIgURE 8: Structure diagram of the test bench.

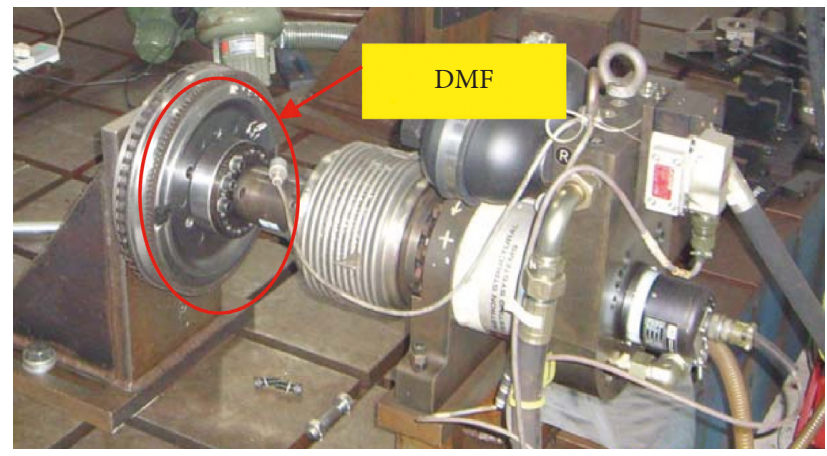

FIGURE 9: DMF test bench.

TABle 1: Parameters of DMF.

\begin{tabular}{lccccc}
\hline Symbol & $J_{1}$ & $J_{2}$ & $M_{0}$ & $k$ & $\theta_{\text {max }}$ \\
\hline Parameter & First mass inertia & Second mass inertia & Frictional disc torque & Arc spring stiffness & Maximum angle \\
Value & $0.121 \mathrm{~kg} \cdot \mathrm{m}^{2}$ & $0.036 \mathrm{~kg} \cdot \mathrm{m}^{2}$ & $10 \mathrm{~N} \cdot \mathrm{m}$ & $3.7 \mathrm{~N} \cdot \mathrm{m} /{ }^{\circ}$ & $60^{\circ}$ \\
\hline
\end{tabular}

(ii) The actuator applied static load $\Theta=20^{\circ}$ to the DMF, and then dynamic load $\theta_{0} \sin \omega t$ is applied, where $\theta_{0}=2.5^{\circ}$ and $\omega=1 \mathrm{~Hz}$

(iii) The angle and torque data were recorded by using a data acquisition device

(iv) The dynamic stiffness of the corresponding excitation is calculated by the geometric mapping method

(v) Change the excitation frequency and repeat steps 1-4 to get dynamic stiffness at different frequencies

(vi) Change $\theta_{0}=3.5^{\circ}$ or $\theta_{0}=5^{\circ}$ and repeat steps $1-5$ to get dynamic stiffness at different magnitude of the excitation angle

The detailed test combinations are shown in Table 2.

4.2. Dynamic Torsion Performance of DMF. The dynamic torsion characteristics of DMF are mainly decided by using the complex stiffness $k_{T}$, dynamic stiffness $k_{\mathrm{d}}$, and lag angle $\psi$. Referring to the standard of SAE (1085a), the complex stiffness $k_{T}$ is the ratio between the transfer torque and the rotation angle of the DMF, which is the vector sum of the elastic component (dynamic stiffness $k_{\mathrm{d}}$ ) and the damping component $\left(C_{\omega}\right) \cdot k_{\mathrm{d}}$ is the projection of $k_{T}$ in the direction of rotation angle. $\psi$ is the angle between complex stiffness $k_{T}$ and the direction rotation angle. Figure 10 is a geometric drawing method for calculating the transfer complex stiffness, dynamic stiffness, and damping lag angle [18-20]. The specific calculation method is as follows:

$$
\left|k_{T}\right|=\frac{2 T_{0}}{2 \theta_{0}}
$$

where $T_{0}$ is the torque amplitude delivered to the second mass and $\theta_{0}$ is the magnitude of the excitation torsion angle:

$$
\begin{aligned}
k_{\mathrm{d}} & =\frac{2 T_{0}}{2 \theta_{0}} \cos \psi, \\
\sin \psi & =\frac{2 S_{1}}{\pi S_{2}},
\end{aligned}
$$

where $S_{1}$ is the area occupied by the ellipse in Figure 11 and $S_{2}$ is one-half of the area of the rectangle ABCD in Figure 11.

4.3. Model Parameter Identification and Verification. The parameters of the DMF dynamic characteristics model in this paper can be divided into two categories. One has clear physical meaning and can be given directly. They are shown 
TABLE 2: Loading scheme for the dynamic torsion test of DMF.

\begin{tabular}{lccc}
\hline Pretwist $\Theta\left({ }^{\circ}\right)$ & 20 & 20 & 20 \\
\hline Amplitude $\theta_{0}\left({ }^{\circ}\right)$ & 2.5 & 3.5 & 5 \\
Sweep frequency range $(\mathrm{Hz})$ & $1-30$ & $1-30$ & $1-30$ \\
\hline
\end{tabular}

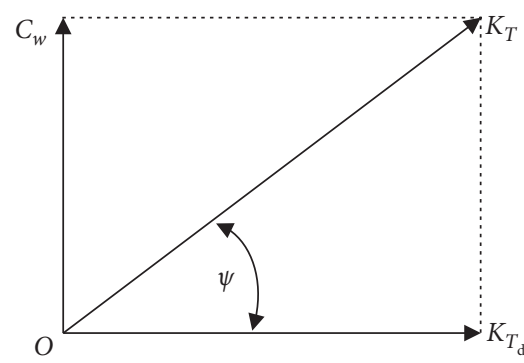

Figure 10: Dynamic characteristic parameter diagram.

in Table 3. The other kind of parameters should be identified by the model parameter identification method because it has no clear physical meaning.

It should be noted that the effect of centrifugal force on DMF is not taken into account in the dynamic torsion test of DMF. It is because that the test equipment cannot be dynamically loaded under the high-speed rotation condition. Only the dynamic loading at $0 \mathrm{rpm}$ can be performed, so the parameter $c_{\text {ef } 2}=0$.

In the process of parameter identification, the optimization objective is set as the sum of the relative error values of the dynamic characteristic curve before and after optimization. The objective function is

$$
\text { opt }=f_{\min }(x)=\left[\frac{k_{\mathrm{de}}-k_{\mathrm{ds}}}{k_{\mathrm{de}}}\right]^{2}+\left[\frac{\psi_{\mathrm{de}}-\psi_{\mathrm{ds}}}{\psi_{\mathrm{de}}}\right]^{2},
$$

where $x$ is the optimization variable, $k_{\mathrm{de}}$ and $k_{\mathrm{ds}}$ are the dynamic stiffness values obtained by experiment and simulation, respectively, and $\psi_{\mathrm{de}}$ and $\psi_{\mathrm{ds}}$ are the lag angle values obtained by experiment and simulation, respectively.

Optimization variable is defined as

$$
x=\left[\begin{array}{llll}
b & \beta & c_{\mathrm{eq}} & c_{\mathrm{e}}
\end{array}\right] \text {. }
$$

In this paper, the Matlab genetic algorithm toolbox is used for parameter identification. The results are shown in Table 4.

The experimental results are compared as shown in Figures 12-14.

The comparison of dynamic stiffness and lag angle results of the model with the results of the test is shown in Figures 12-14. The dynamic stiffness and lag angle results of the model and test match well. Thus, the reliability of the model can be verified. Figures 12-14 show that the resonance of DMF occurs near $10 \mathrm{~Hz}$. The dynamic stiffness of the DMF is the smallest, and the damping angle is $90^{\circ}$. The idle speed of an automobile engine is generally $750 \mathrm{rpm}$, and the corresponding ignition frequency of the engine is $25 \mathrm{~Hz}$, which far exceeds the resonance frequency of the DMF, so the powertrain does not resonate during the normal working speed of the vehicle. But the engine must pass through the

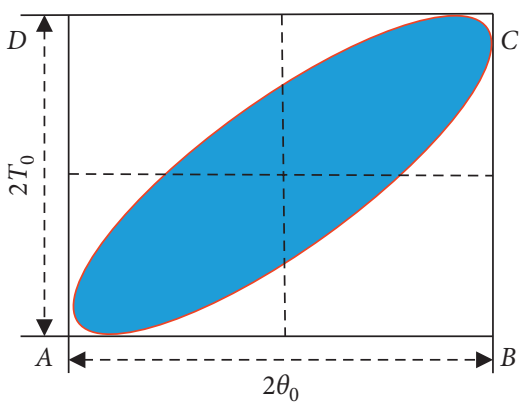

Figure 11: Hysteresis loop diagram.

resonance speed of the DMF in the ignition process, and resonance will occur at this time; due to the short ignition time, the problem of NVH will not be serious. At the same time, the frictional damping generated by using the friction disc will greatly attenuate the torsional vibration of the DMF resonance.

4.4. Model Error Analysis. In this section, the error is reflected by the difference between the calculated value and the experimental value of the model. The curve of the model error with frequency is shown in Figures 15-17.

As shown in Figures 15-17, the errors of dynamic stiffness and lag angle of the model increase first and then decrease with the increase of frequency. Besides, the maximum error occurs near the resonance frequency. When the excitation frequency is greater than the natural frequency, the model error decreases rapidly as the frequency increases.

The average error percentages of dynamic stiffness and lag angle are $18.8 \%$ and $10.3 \%$, respectively. It is found that when the DMF is near the resonance frequency point, the minimum dynamic stiffness value is only $1.3 \mathrm{~N} \cdot \mathrm{m} /{ }^{\circ}$, while the calculation result of the model is $2.08 \mathrm{~N} \cdot \mathrm{m} /{ }^{\circ}$, and the error is as high as $60 \%$. This is the reason for the larger average error. The resonance state is considered as abnormal working state, and the normal working frequency of the DMF is generally greater than $20 \mathrm{~Hz}$. Therefore, only the percentage of the model error above $20 \mathrm{~Hz}$ is calculated, which is $4.97 \%$ and $1.19 \%$, respectively. The accuracy of the model meets the engineering requirements. The reason for the large error of resonance frequency may be that the arc spring is replaced by equivalent mass.

\section{DMF Dynamic Characteristics Test Results}

In this section, frequency dependency characteristics and amplitude dependency characteristics of DMF are experimentally studied by comparing different pretwisted angles and excitation angles amplitude (Table 5).

5.1. Same Pretwisted Angle $\Theta$, Different Excitation Amplitudes $\theta_{0}$. Figures 18 and 19 show the influence of excitation frequency on dynamic stiffness $k_{\mathrm{d}}$ and lag angle $\psi$ of DMF under different excitation amplitudes when the pretwisted angle $\Theta$ is constant. It can be seen that the dynamic stiffness $k_{\mathrm{d}}$ and lag angle $\psi$ of the DMF are greatly affected by the 
TABLE 3: Known parameters.

\begin{tabular}{lccccc}
\hline Symbol & $m_{\mathrm{e}}$ & $R$ & $C$ & $k_{\mathrm{e}}$ & $p_{1}$ \\
\hline Parameter & Arc spring mass & Distribution radius & Damping coefficient & Arc spring stiffness & Fitting coefficient \\
Value & $0.6 \mathrm{~kg}$ & $0.12 \mathrm{~m}$ & 0.02 & $6.7 \mathrm{~N} \cdot \mathrm{m} / /^{\circ}$ & 0.5619 \\
\hline
\end{tabular}

TABLE 4: Identification results.

\begin{tabular}{lcccc}
\hline Symbol & $b$ & $\beta$ & $c_{\mathrm{eq}}$ & $c_{\mathrm{e}}$ \\
\hline Parameter value & 0.12 & 1.9 & 1.579 & 0.56 \\
\hline
\end{tabular}

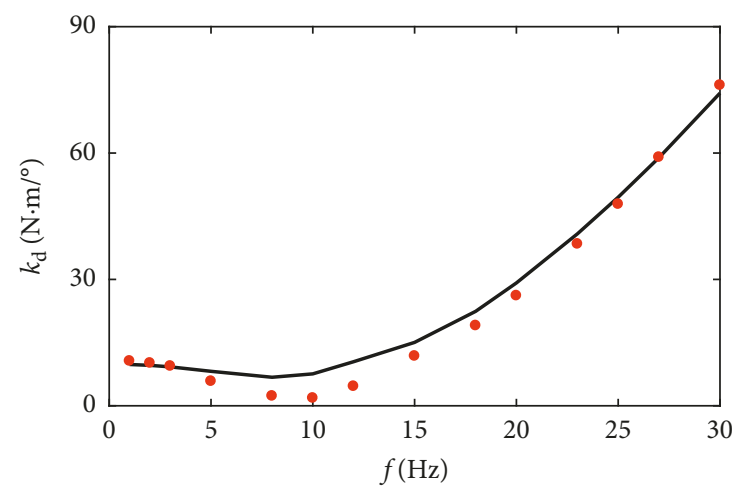

- Model

- Experiment

(a)

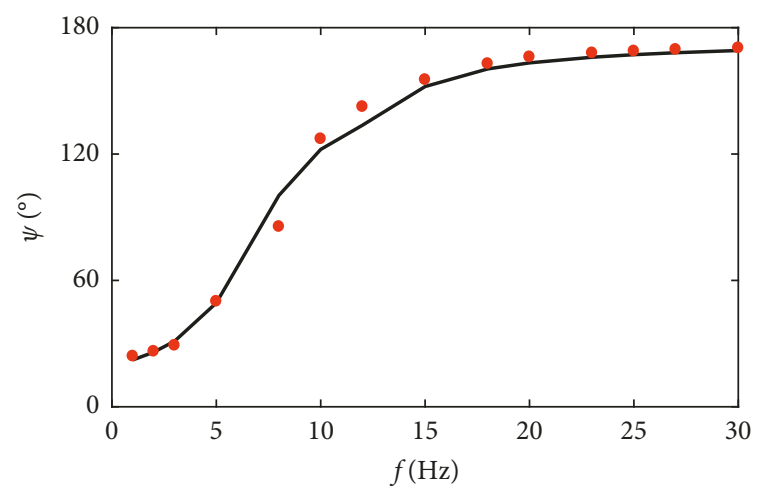

- Model

- Experiment

Figure 12: Excitation amplitude $\theta_{0}=2.5^{\circ}$. (a) Dynamic stiffness $k_{\mathrm{d}}$. (b) Lag angle $\psi$.

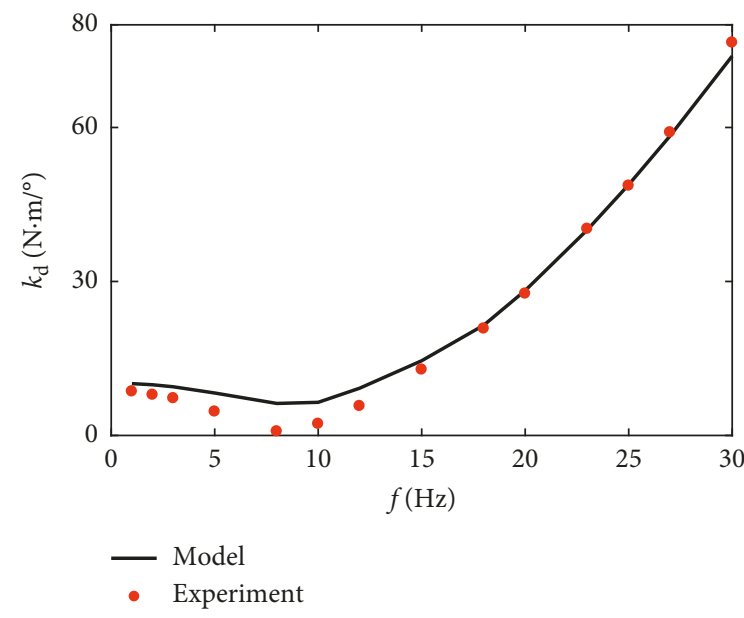

(a)

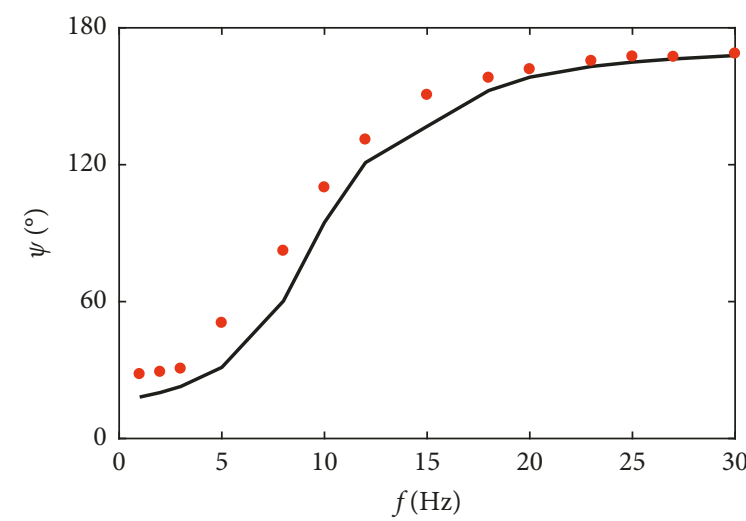

- Model
Experiment

(b)

Figure 13: Excitation amplitude $\theta_{0}=3.5^{\circ}$. (a) Dynamic stiffness $k_{\mathrm{d}}$. (b) Lag angle $\psi$.

excitation frequency. The dynamic stiffness $k_{\mathrm{d}}$ tends to decrease first and then increase with the increase of the excitation frequency and reaches a minimum near $9 \mathrm{~Hz}$. Because the resonance frequency of the DMF is about $10 \mathrm{~Hz}$, the dynamic stiffness reaches the minimum at this time. When the pretwist is the same, the change of the excitation amplitude $\theta_{0}$ causes the dynamic stiffness $k_{\mathrm{d}}$ characteristic curve shift to the left. Therefore, when the excitation frequency is less than $10 \mathrm{~Hz}$, the dynamic stiffness $k_{\mathrm{d}}$ decreases with the increase of excitation amplitude $\theta_{0}$. When the excitation frequency is greater than $10 \mathrm{~Hz}$, the dynamic stiffness $k_{\mathrm{d}}$ increases with the increase of the excitation amplitude $\theta_{0}$. But in general, the change in the excitation amplitude $\theta_{0}$ has less effect on the dynamic stiffness $k_{\mathrm{d}}$. 


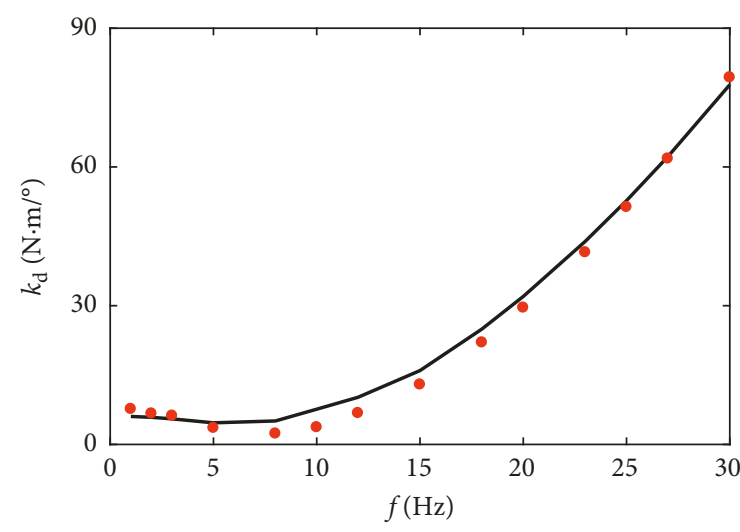

- Model

- Experiment

(a)

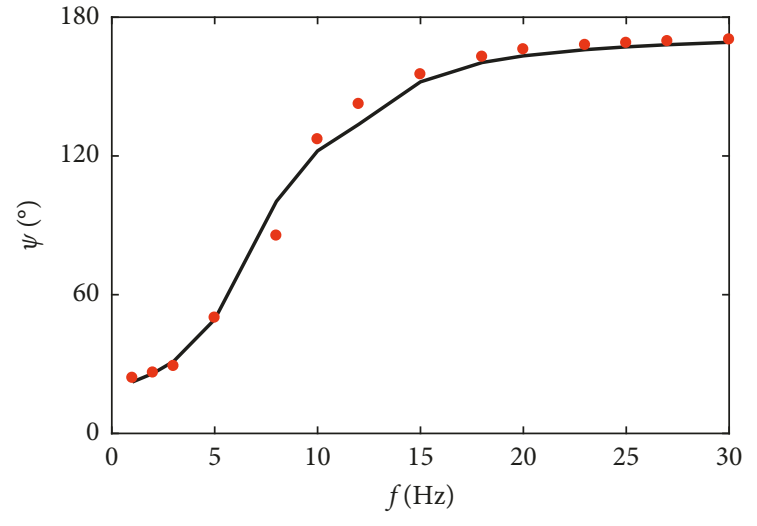

- Model
Experiment

Figure 14: Excitation amplitude $\theta_{0}=5^{\circ}$. (a) Dynamic stiffness $k_{\mathrm{d}}$. (b) Lag angle $\psi$.

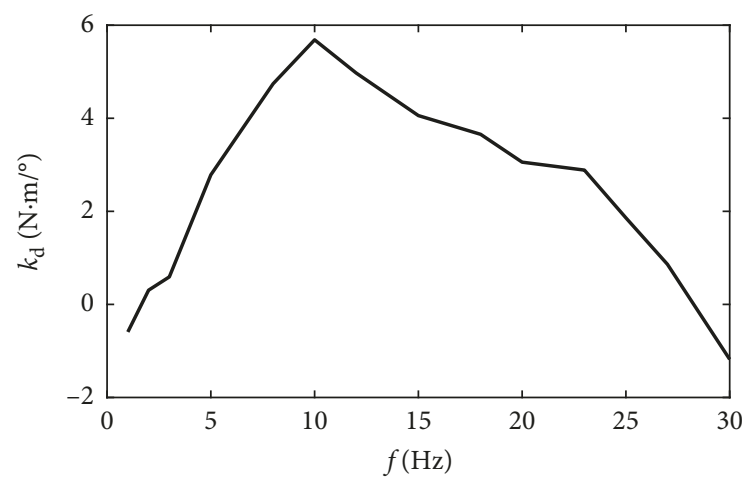

(a)

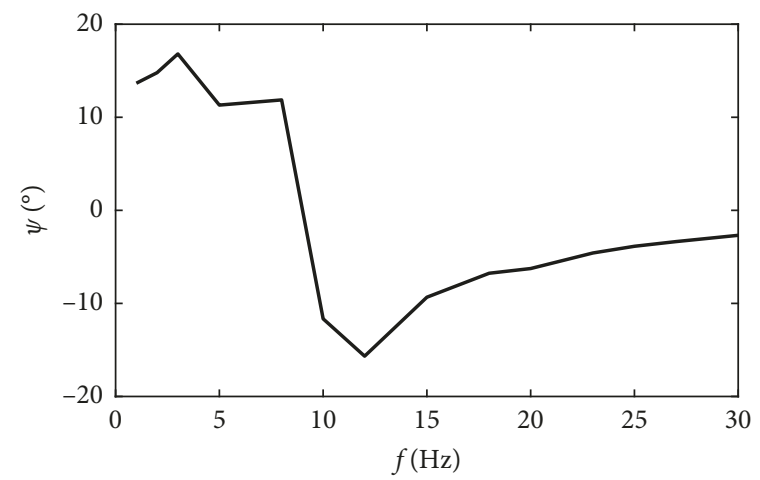

(b)

FIgURE 15: Model error curve with excitation amplitude of $\theta_{0}=2.5^{\circ}$. (a) Dynamic stiffness error $k_{\mathrm{d}}$. (b) Lag angle error $\psi$.

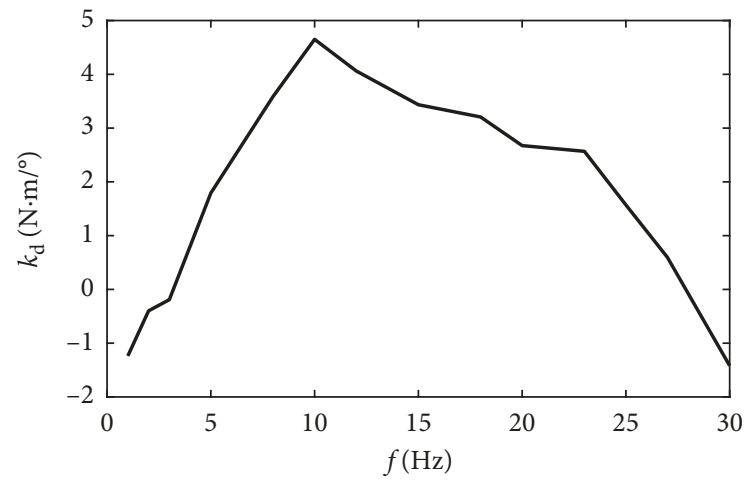

(a)

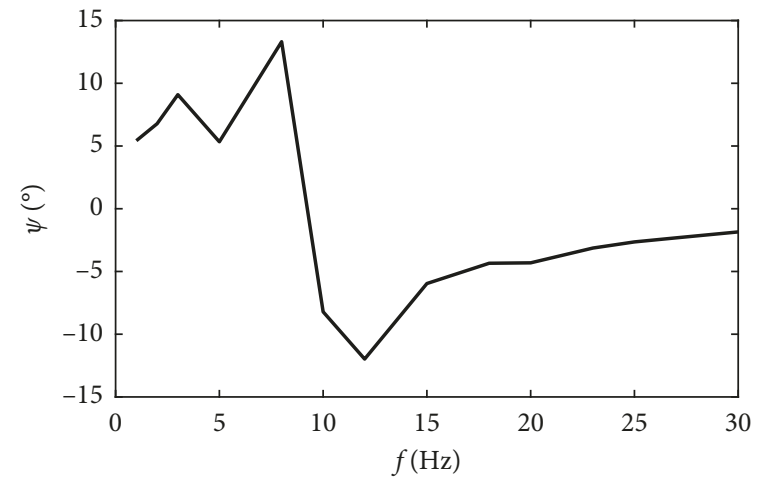

(b)

FIgURE 16: Model error curve with excitation amplitude of $\theta_{0}=3.5^{\circ}$. (a) Dynamic stiffness error $k_{\mathrm{d}}$. (b) Lag angle error $\psi$.

The lag angle $\psi$ increases as the excitation frequency increases, reaching 90 degrees near $10 \mathrm{~Hz}$. When the excitation frequency exceeds $20 \mathrm{~Hz}$, the lag angle $\psi$ tends to a stable value. When the excitation amplitude $\theta_{0}$ increases, the characteristic curve of the lag angle $\psi$ shifts to the left. The frequency value corresponding to the $90^{\circ}$ value becomes smaller, and the stability value of the lag angle $\psi$ also becomes larger. 


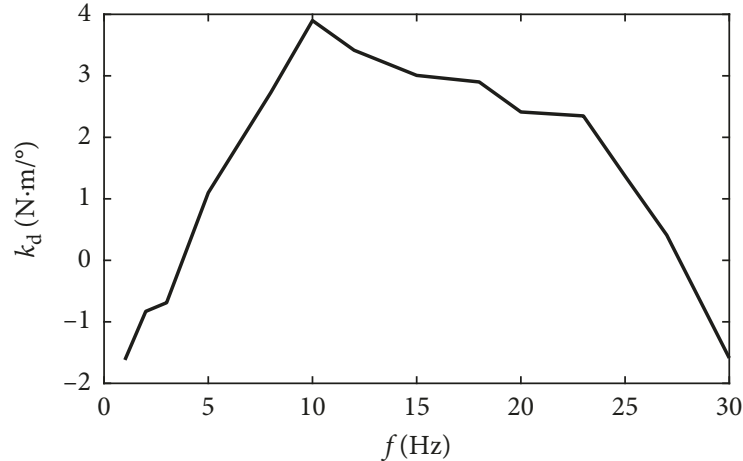

(a)

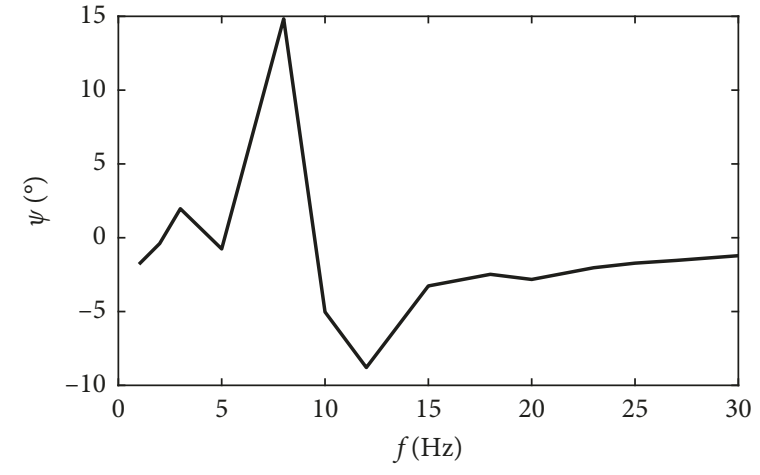

(b)

Figure 17: Model error curve with excitation amplitude of $\theta_{0}=5^{\circ}$. (a) Dynamic stiffness error $k_{\mathrm{d}}$. (b) Lag angle error $\psi$.

TABLE 5: Loading scheme.

\begin{tabular}{lcc}
\hline Pretwist $\Theta\left({ }^{\circ}\right)$ & 5 & 20 \\
\hline Amplitude $\theta_{0}\left({ }^{\circ}\right)$ & $2.5,3.5,5$ & $2.5,3.5,5$ \\
Sweep frequency range $f(\mathrm{~Hz})$ & $1-30$ & $1-30$ \\
\hline
\end{tabular}

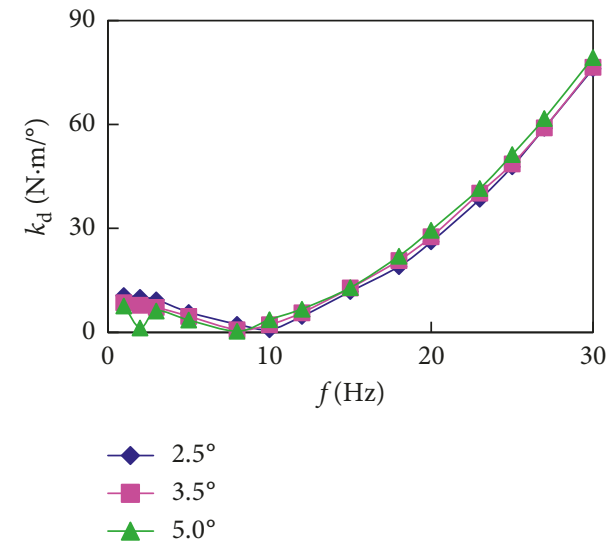

(a)

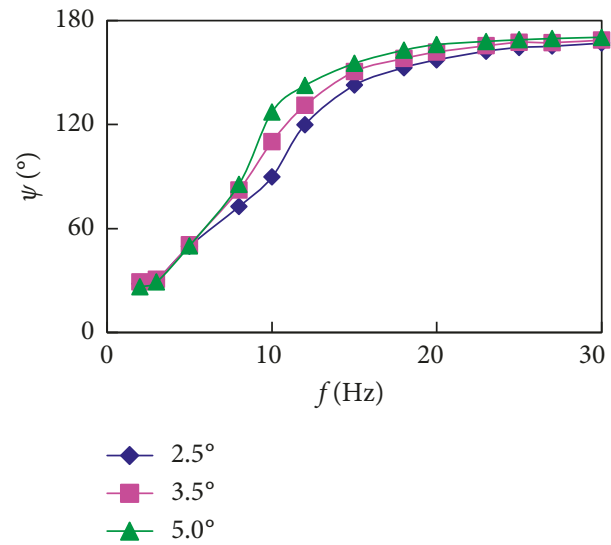

(b)

Figure 18: Different excitation amplitudes at $\Theta=5^{\circ}$. (a) Dynamic stiffness $k_{\mathrm{d}}$. (b) Lag angle $\psi$.

5.2. Same Excitation Amplitude $\theta_{0}$, Different Pretwisted $\Theta$. Figures $20-22$ show the influence of the excitation frequency on the dynamic stiffness $k_{\mathrm{d}}$ and the lag angle $\psi$ of the DMF. At different pretwisted angles $\Theta$ when the excitation amplitude $\theta_{0}$ is constant. It can be seen from the figure that the dynamic stiffness $k_{\mathrm{d}}$ of the DMF decreases with the increase of the pretwisted angle $\Theta$ under the same excitation amplitude $\theta_{0}$, especially when the excitation frequency is greater than $10 \mathrm{~Hz}$. Under the same excitation amplitude $\theta_{0}$, the lag angle $\psi$ also increases as the pretwisted angle $\Theta$ increases, and the stable value of the lag angle $\psi$ decreases as the pretwisted angle $\Theta$ increases.

The torsional characteristics test results of the DMF show that the dynamic torsional characteristics have obvious nonlinear characteristics, mainly in the aspects of frequency correlation and amplitude correlation. The torsional characteristics of the DMF determine its damping performance.
Therefore, frequency variation and variation characteristics need to be fully considered in the process of structure design and parameter matching of the DMF.

\section{Conclusion}

In this paper, theoretical modeling and experimental verification of dynamic torsion characteristics of DMF are carried out:

(1) Based on the influence of the spring frictional torque and other factors on the dynamic torsion characteristics of DMF, the nonlinear dynamic torsional vibration model of the DMF is established by using the discrete element method, equivalent energy method, and fractional derivative. The model is verified by experiments, and the results show that the 


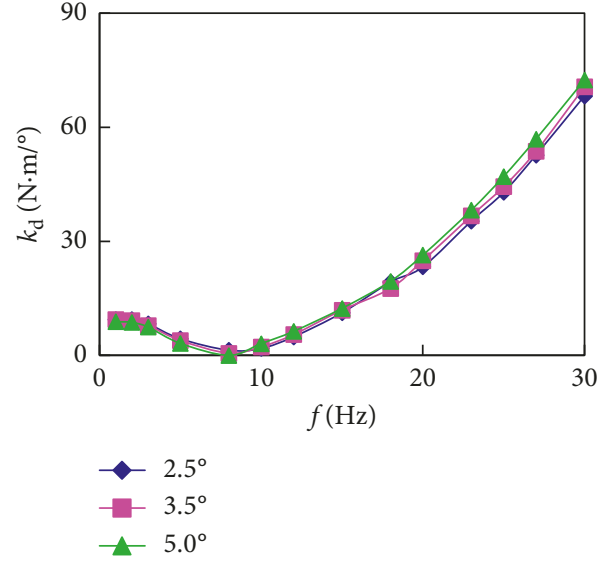

(a)

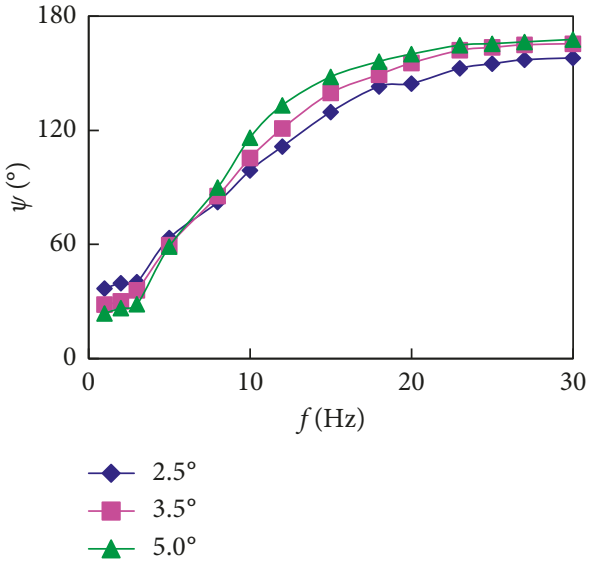

(b)

Figure 19: Different excitation amplitudes at $\Theta=20^{\circ}$. (a) Dynamic stiffness $k_{\mathrm{d}}$. (b) Lag angle $\psi$.

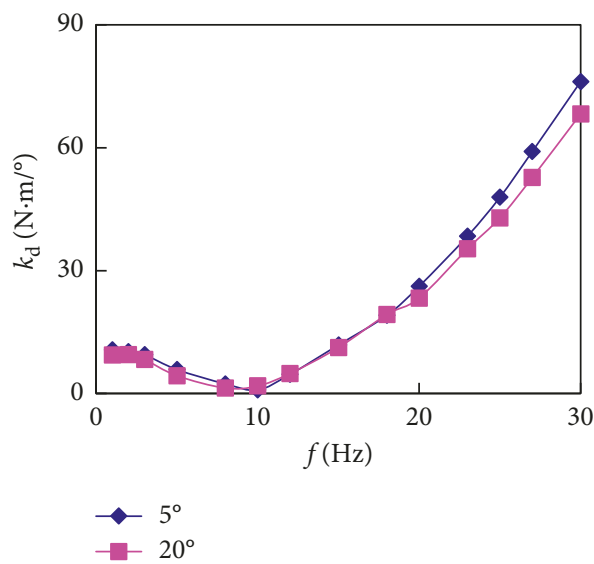

(a)

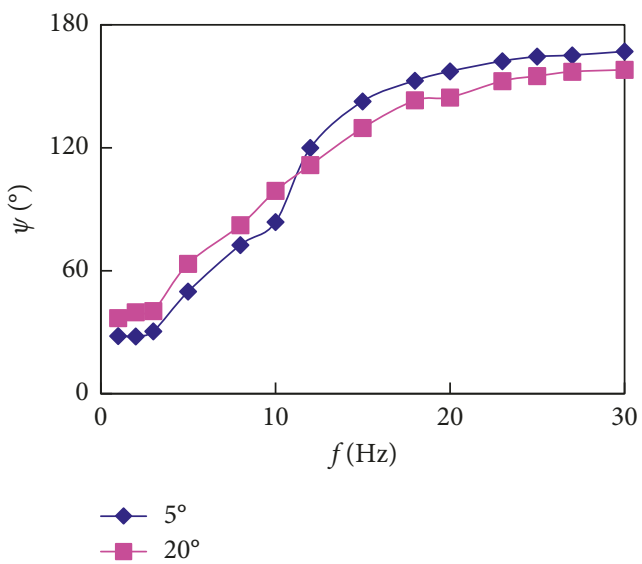

(b)

Figure 20: Different pretwisted angles at $\theta_{0}=2.5^{\circ}$. (a) Dynamic stiffness $k_{\mathrm{d}}$. (b) Lag angle $\psi$.
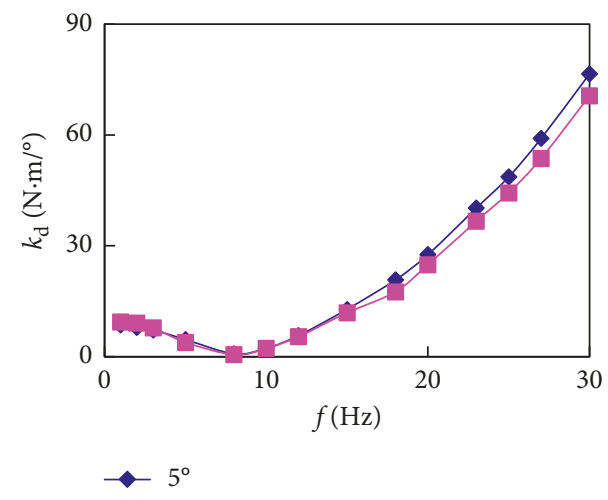

$$
-20^{\circ}
$$

(a)

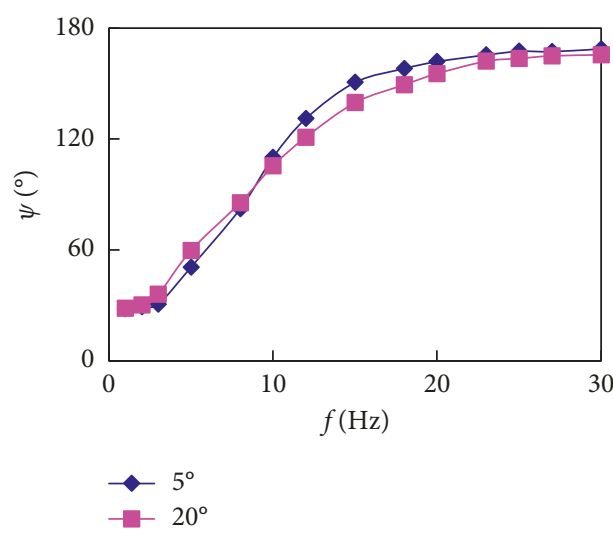

(b)

Figure 21: Different pretwisted angles at $\theta_{0}=3.5^{\circ}$. (a) Dynamic stiffness $k_{\mathrm{d}}$. (b) Lag angle $\psi$. 


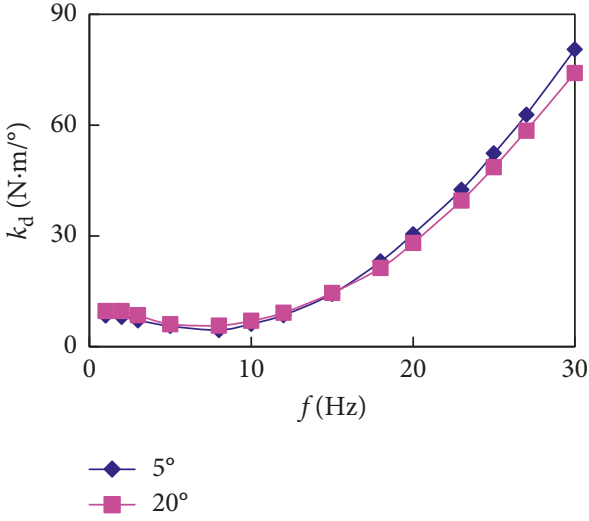

(a)

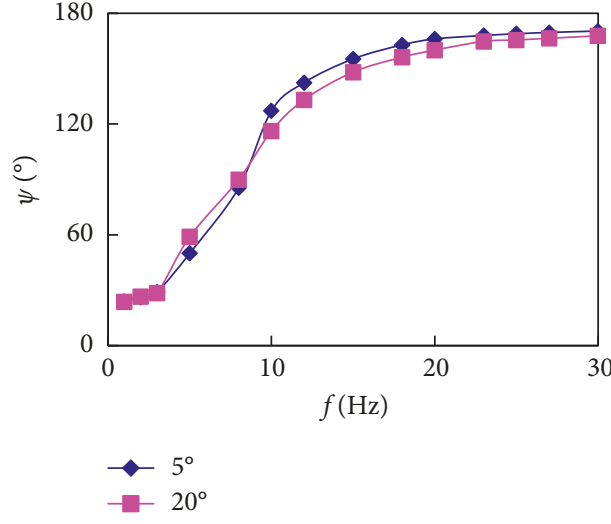

(b)

Figure 22: Different pretwisted angles at $\theta_{0}=5^{\circ}$. (a) Dynamic stiffness $k_{\mathrm{d}}$. (b) Lag angle $\psi$.

accuracy of the model meets the engineering requirements.

(2) Through the error analysis of the nonlinear dynamic characteristic model of the DMF, the maximum error occurs near the resonance frequency. However, the result is not important, considering that the resonance state is abnormal and the working frequency of the DMF is more than $20 \mathrm{~Hz}$. The model error of the common working frequency is less than $5 \%$, which meets the engineering requirements.

(3) Through the experimental study on the dynamic torsional characteristics of the DMF, it is found that the dynamic stiffness and the lag angle have significant nonlinear characteristics, and its performance is related to the excitation frequency and pretwisted angle. It is necessary to consider the effect of nonlinear torsional characteristics on the damping performance in the process of structure design and parameter matching.

\section{Nomenclature}

$\theta: \quad$ Torsion angle of DMF

T: $\quad$ Torsion of DMF

$\Omega$ : $\quad$ Rotating speed of DMF

$m: \quad$ Total mass of arc spring

$m_{\mathrm{e}}: \quad$ Equivalent mass

$n: \quad$ The number of units

$m_{i}: \quad$ The unit mass of DMF

$k_{i}$ : The unit stiffness of DMF

$k_{\mathrm{e}}$ : $\quad$ Equivalent stiffness

$c_{i}$ : The unit damping coefficient of DMF

$\theta_{i}: \quad$ The angle between elements

$F_{k-1}$ : The force of the last spring mass element

$F_{k}$ : The force of the next spring mass element

$F_{\mathrm{F}}$ : The tangential friction force

$F_{0}$ : $\quad$ The radial force

$F_{N}$ : The support force

$\mu$ : $\quad$ Friction coefficient

$\alpha: \quad$ The central angle of the arc spring's free length $\varphi_{i}: \quad$ The angle difference of any two elements

$T_{\mathrm{f} 1}$ : $\quad$ Frictional torque generated by radial component

$T_{\mathrm{f} 2}$ : $\quad$ Frictional torque generated by centrifugal force

$T_{\mathrm{f}}$ : $\quad$ Total frictional torque $T_{\mathrm{f} 1}$ and $T_{\mathrm{f} 2}$ of arc spring

$T_{\text {fri }}: \quad$ Frictional torque of the friction disc

$c, c_{\mathrm{e} 1}, c_{\mathrm{eq}}$ : The equivalent damping coefficient

$R: \quad$ Arc spring distribution radius

$\theta_{0}: \quad$ Excitation amplitude

$\omega: \quad$ Excitation frequency

$T_{\mathrm{s}}$ : $\quad$ The recovery torque of the spring

$T_{\mathrm{d}}$ : The viscous damping torque

$b$ : $\quad$ The viscoelastic torque coefficient

$\beta$ : $\quad$ Order derivative

$k_{\mathrm{d}}: \quad$ Dynamic stiffness

$\psi$ : $\quad$ Lag angle

$\Theta: \quad$ Pretwist

\section{Data Availability}

The data used to support the findings of this study are included within the article.

\section{Conflicts of Interest}

The authors declare that there are no conflicts of interest regarding the publication of this paper.

\section{Acknowledgments}

The authors acknowledge the financial support from the National Key R\&D Program of China (no. 2018YFB0106203).

\section{References}

[1] A. Albers, "Advanced development of dual mass flywheel (DMFW) design-noise control for today's automobiles," in Proceedings of the 5th LuK Symposium, pp. 5-41, Bühl, Germany, May 1994.

[2] R. Wolfgang, S. Roland, and A. Kooy, "Dual mass flywheel," in Proceedings of the 6th LuK Symposium, Bühl, Germany, 1998. 
[3] A. Fidlin and R. Seebacher, "DMF simulation techniques," in Proceedings of the 8th LuK Symposium, pp. 55-71, Bühl, Germany, 2006.

[4] T. H. Kim, H. L. Song, S. H. Hwang, and H. S. Kim, "Analysis of dual mass flywheel using discrete arcspring model," Key Engineering Materials, vol. 326-328, pp. 1607-1610, 2006.

[5] U. Schaper, O. Sawodny, T. Mahl, and U. Blessing, "Modeling and torque estimation of an automotive dual mass flywheel," in Proceedings of the American Control Conference (ACC'09), pp. 1207-1212, IEEE, St. Louis, MO, USA, June 2009.

[6] L. Chen, R. Zeng, and Z. Jiang, "Nonlinear dynamical model of an automotive dual mass flywheel," Advances in Mechanical Engineering, vol. 7, no. 6, article 1687814015589533, 2015.

[7] L. Q. Song, L. P. Zeng, S. P. Zhang, J. D. Zhou, and H. E. Niu, "Design and analysis of a dual mass flywheel with continuously variable stiffness based on compensation principle," Mechanism and Machine Theory, vol. 79, pp. 124-140, 2014.

[8] L. P. Zeng, L. Q. Song, and J. D. Zhou, "Design and elastic contact analysis of a friction bearing with shape constraint for promoting the torque characteristics of a dual mass flywheel," Mechanism and Machine Theory, vol. 92, pp. 356-374, 2015.

[9] Q.-H. Zu, Z.-Y. Chen, W.-K. Shi, Y. Mao, and Z.-Y. Chen, "Torsional vibration semiactive control of drivetrain based on magnetorheological fluid dual mass flywheel," Mathematical Problems in Engineering, vol. 2015, Article ID 608737, 17 pages, 2015.

[10] X. Tang, X. Hu, W. Yang, and H. Yu, "Novel torsional vibration modeling and assessment of a power-split hybrid electric vehicle equipped with a dual-mass flywheel," IEEE Transactions on Vehicular Technology, vol. 67, no. 3, pp. 1990-2000, 2018.

[11] X. Tang, D. Zhang, T. Liu, A. Khajepour, H. Yu, and H. Wang, "Research on the energy control of a dual-motor hybrid vehicle during engine start-stop process," Energy, vol. 166, pp. 1181-1193, 2019.

[12] X. Tang, W. Yang, X. Hu, and D. Zhang, "A novel simplified model for torsional vibration analysis of a series-parallel hybrid electric vehicle," Mechanical Systems and Signal Processing, vol. 85, pp. 329-338, 2017.

[13] Y. Qin, C. Wei, X. Tang, N. Zhang, M. Dong, and C. Hu, "A novel nonlinear road profile classification approach for controllable suspension system: simulation and experimental validation," Mechanical Systems and Signal Processing, vol. 125, pp. 79-98, 2019.

[14] Z. F. Yan and Z. F. Jiang, "Research on the integrated design of dual mass flywheel CAD/CAE/CAM," Key Engineering Materials, vol. 407-409, pp. 169-173, 2009.

[15] S. Theodossiades, M. Gnanakumarr, H. Rahnejat, and P. Kelly, "Effect of a dual-mass flywheel on the impact-induced noise in vehicular powertrain systems," Proceedings of the Institution of Mechanical Engineers, Part D: Journal of Automobile Engineering, vol. 220, no. 6, pp. 747-761, 2006.

[16] J. P. Den Hartog, Mechanical Vibrations, Courier Corporation, Chelmsford, MA, USA, 1985.

[17] K. Sedlaczek, S. Dronka, and J. Rauh, "Advanced modular modelling of rubber bushings for vehicle simulations," Vehicle System Dynamics, vol. 49, no. 5, pp. 741-759, 2011.

[18] J. E. Colgate, C.-T. Chang, Y.-C. Chiou, W. K. Liu, and L. M. Keer, "Modelling of a hydraulic engine mount focusing on response to sinusoidal and composite excitations," Journal of Sound and Vibration, vol. 184, no. 3, pp. 503-528, 1995.

[19] W.-K. Shi, Q. Chen, Z.-Y. Chen, C. Yang, and H.-H. Zhang, "Modeling and dynamic properties of a four-parameter Zener model vibration isolator," Shock and Vibration, vol. 2016, Article ID 5081812, 16 pages, 2016.

[20] A. K. Mallik, V. Kher, M. Puri, and H. Hatwal, "On the modelling of non-linear elastomeric vibration isolators," Journal of Sound and Vibration, vol. 219, no. 2, pp. 239-253, 1999. 


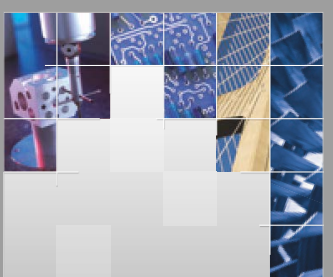

\section{Enfincering}
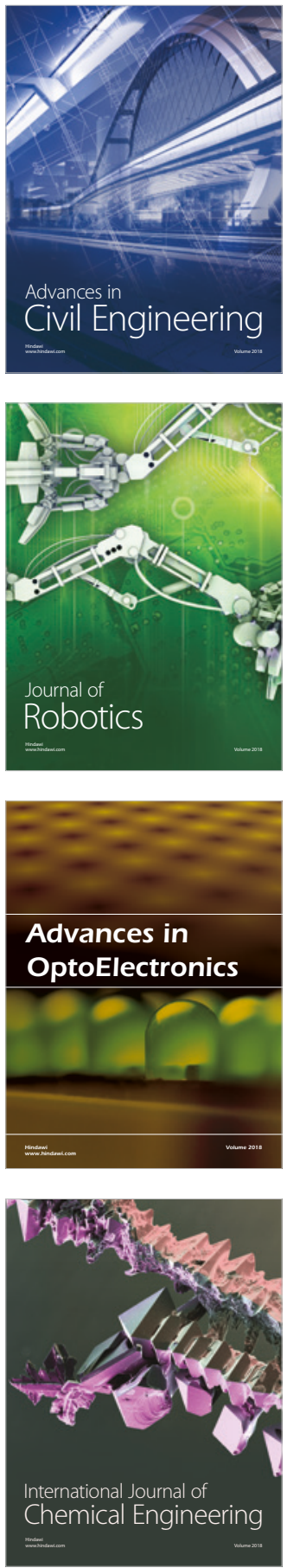

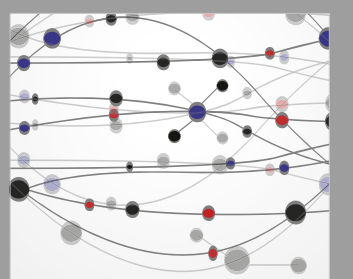

\section{Rotating \\ Machinery}

The Scientific World Journal

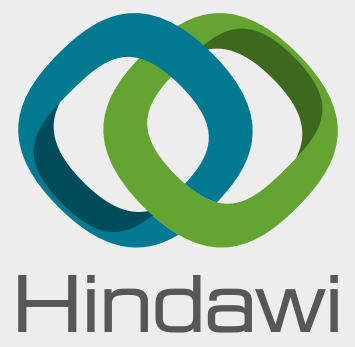

Submit your manuscripts at

www.hindawi.com
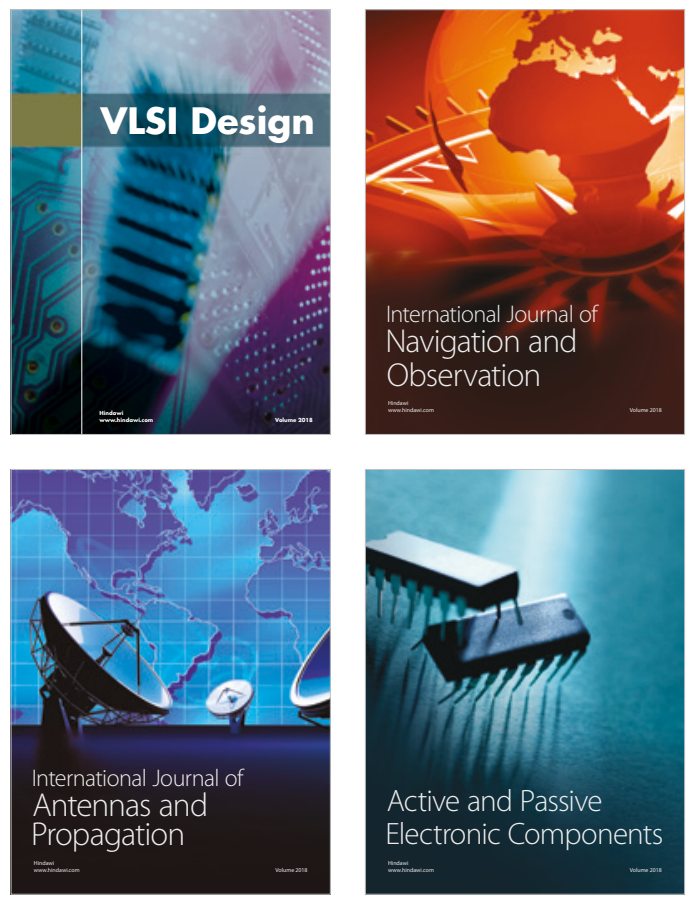
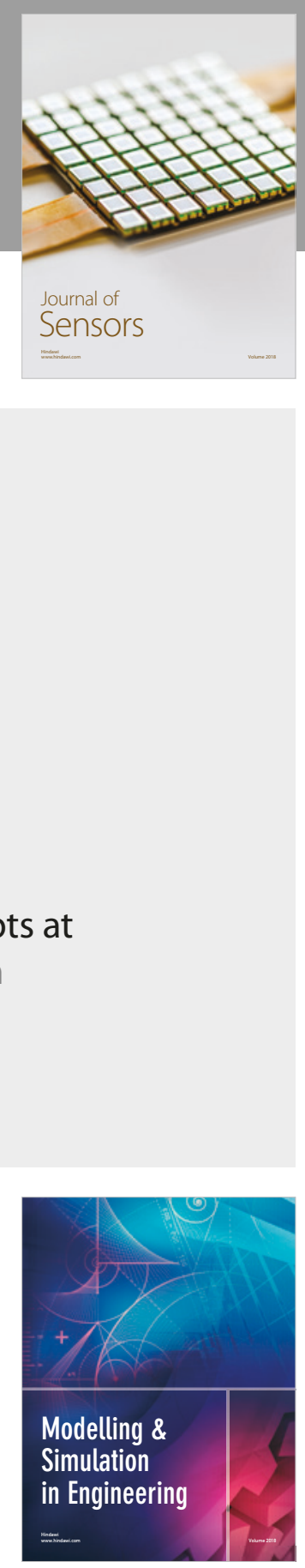

\section{Advances \\ Multimedia}
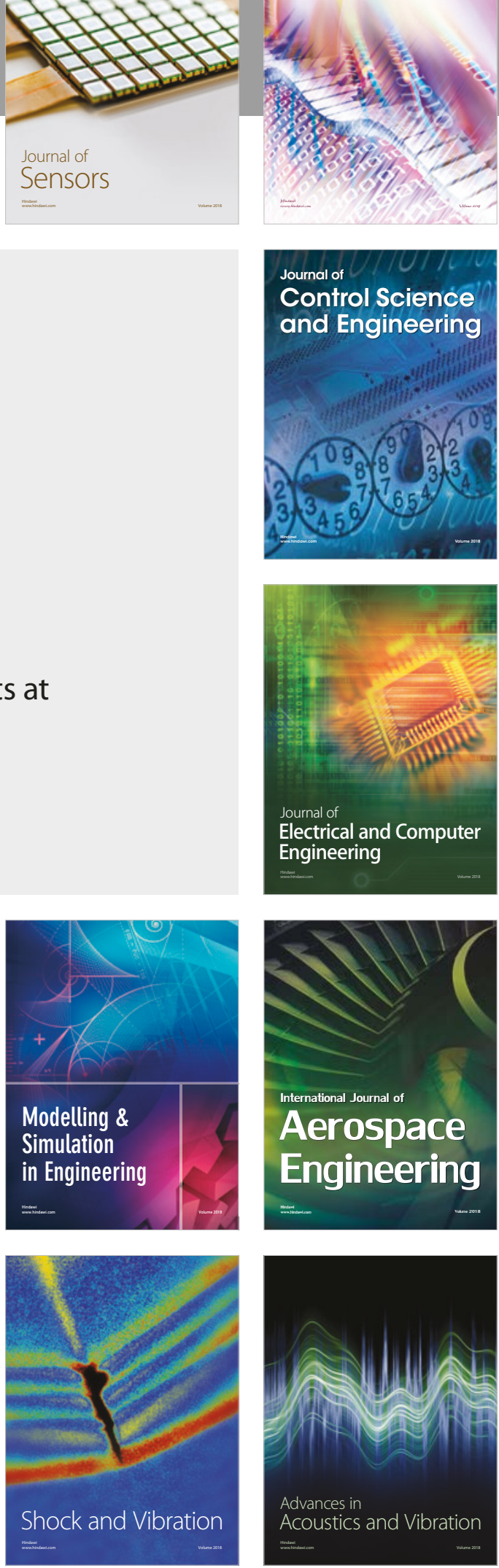